\title{
Structural and electronic properties of ultrathin picene films on the $\operatorname{Ag}(100)$ surface
}

Simon J. Kelly ${ }^{1}$, Dan C. Sorescu ${ }^{2,3}$, Jun Wang ${ }^{1}$, Kaye A. Archer ${ }^{4}$, Kenneth D. Jordan ${ }^{3,4}$ and Petro Maksymovych $^{1}$

${ }^{1}$ Center for Nanophase Materials Sciences, Oak Ridge National Laboratory (ORNL), United States

${ }^{2}$ National Energy Technology Laboratory, U.S. Department of Energy, Pittsburgh, Pennsylvania 15236, United States

${ }^{3}$ Department of Chemical and Petroleum Engineering, University of Pittsburgh, Pittsburgh, Pennsylvania, 15261, United States

${ }^{4}$ Department of Chemistry, University of Pittsburgh, Pittsburgh, Pennsylvania 15260, United States

Keywords: picene, tunneling spectroscopy, potassium, silver, electron affinity 


\begin{abstract}
Using scanning tunneling microscopy and electronic structure calculations, we investigated the assembly and electronic properties of picene molecules on the $\operatorname{Ag}(100), \operatorname{Ag}(111)$, and $\mathrm{Cu}(111)$ surfaces, with particular emphasis on $\operatorname{Ag}(100)$. In each case, picene molecules are found to lie parallel to the surface at coverages up to half a monolayer and to adopt alternating parallel and tilted orientations at full monolayer coverage. In the latter case, the arrangement of the molecules is roughly similar to that in the bulk crystal, making ultrathin films of picene interesting model systems and worthwhile to explore its electronic properties. On the metal surfaces considered, the growth mode of picene is quite different from that of its structural isomer pentacene, which forms bilayer coverage on top of a dense monolayer of flat-lying molecules on metal surfaces. Tunneling spectroscopy measurements provide estimates of the energies of several low-lying molecular orbitals as well as of the highest unoccupied molecular orbital of the absorbed picene molecules. From analysis of these results, we establish that the onsite Coulomb repulsion for picene decreases by $\sim 2 \mathrm{eV}$ in going from the gas phase to the full monolayer on $\operatorname{Ag}(100)$, bringing it close to that of the undoped bulk crystal.
\end{abstract}

\title{
1. Introduction
}

Picene, an isomer of pentacene with an armchair arrangement of benzene rings, has attracted considerable interest for potential applications in electronic materials. Significantly, for the gas-phase molecules, the one-particle band gap is appreciably larger for picene than for pentacene (7.00 vs. $5.35 \mathrm{eV}[1])$ making it more stable. (The band gaps of the corresponding crystals are $3.1 \mathrm{eV}$ for picene [2] and $1.8 \mathrm{eV}$ for pentacene [3]). In addition, it has been reported that the picene crystal, when intercalated with potassium, can become superconducting with a $T_{\mathrm{c}}$ below $7 \mathrm{~K}$ or even as high as $18 \mathrm{~K}$ [4,5], although this result has been questioned by other 
researchers [6]. Following the report of superconductivity in picene, several other PAHs (polycyclic aromatic hydrocarbons) with zig-zag fusion of the rings have been reported to become superconducting when doped with alkali or other metal atoms[5]. Equally intriguing is the nature of the electronic structure of the crystal at high doping levels but under conditions that it is not superconducting [7].

At the same time, evidence is mounting that the doping picture itself is complex. Resistive measurements have revealed that picene undergoes a superconducting transition[8], where the state above $T_{\mathrm{c}}$ is granular-metal-like. Other reports have suggested strong in homogeneities in alkali-doped polyacenes [6,9]. This would be consistent with the inference of filamentary superconductivity where only a fraction of the total sample becomes superconducting [10]. Recent calorimetry measurements indicate that the reported superconductivity in alkali-doped picene may be due to ferromagnetic impurities, or that the molecules may even react with the intercalated $\mathrm{K}$ atoms [6]. In light of the inconsistencies concerning the nature of doped picene films and crystals, it is clear that additional studies providing a molecular level understanding of both the doped and undoped systems are required. Of particular importance for understanding the electronic structure of picene is the extent of electrostatic screening changes in going from the isolated molecule to condensed-phase picene systems $[7,11,12]$.

In this work, we present the first steps toward the development of such picture. Using tunneling microscopy and spectroscopy as well as electronic structure calculations, the monolayer structures of picene on $\operatorname{Ag}(111), \mathrm{Cu}(111)$, and $\operatorname{Ag}(100)$ were characterized. As reported earlier by the Hasegawa group[13], picene on $\operatorname{Ag}(111)$ displays two distinct phases in which all adsorbed molecules are in direct contact with the metal surface. We determined that 
this behavior also occurs for picene on the $\mathrm{Ag}(100)$ and $\mathrm{Cu}(100)$ surfaces. Furthermore, for picene/Ag(100) we determined using tunneling spectroscopy the energies of cation and anion states associated with the highest occupied molecular orbital (HOMO), lowest unoccupied molecular orbital (LUMO), and several higher energy unoccupied states. In a simple orbital model, the HOMO-LUMO gap of a molecule depends linearly on $U$, the on-site Coulomb repulsion[14]. In condensed phase systems, the $U$ value is reduced by screening effects. We determined that the value of $U$ for picene molecule on the metal surface is comparable to that of picene in the bulk crystal. Our first attempt toward intercalation of $\mathrm{K}$ atoms into the picene film, aiming to reduce the nearly neutral adsorbed molecule to an open-shell anion state, introduced very strong disorder into the picene layer, although the procedure used is known to produce wellordered structures of doped $\mathrm{C}_{60}[15]$.

\section{Experimental and computational methods}

\subsection{Experimental}

Picene films were grown in a custom-modified UHV chamber equipped with a deposition source, LEED and a quartz-crystal microbalance. Metal surfaces were cleaned by sputtering/annealing cycles prior to molecular deposition. The molecules were deposited on the metal surfaces at temperatures between 77 and $150 \mathrm{~K}$. Subsequent ordering of the films was achieved by post-annealing to $T=300 \mathrm{~K}$. STM images were acquired and tunneling measurements were carried out with a commercial scanning tunneling microscope (SPECS JTSTM) at $T=77$ and $4.5 \mathrm{~K}$. Tunneling spectroscopy was used to determine the HOMO-LUMO gap as well as the energies of higher-lying anion states of picene on $\operatorname{Ag}(100)$. 


\subsection{Computational Methods}

The adsorption properties of picene molecules on the $\operatorname{Ag}(100)$ surface as a function of coverage were investigated using density functional theory calculations carried out with the Quickstep module [16] of the CP2K program [17]. The calculations made use of the PBE functional [18] with the Grimme-D3 [19] correction for long-range dispersion. The DZVPMOLOPT [20] Gaussian-type orbital (GTO) basis set was used for the Ag atoms, and the TZV2P-MOLOPTGTO basis set was used for the $\mathrm{C}$ and $\mathrm{H}$ atoms of the picene molecule. A plane-wave basis set with a cutoff of 400 Ry was used for expansion of the electronic density.

The binding configurations of the picene molecule at different coverages were investigated using two sets of slab models. The first set of models which included the $(4 \times 4)$, (6x6) and (8x8) square surface models with their axes aligned parallel and perpendicular to the $[0,0.5,-0.5]$ axis was used to characterize the adsorption of picene on the surface at low coverage. The second set used $(7 \times 5)$ and $(8 \times 5)$ rectangular supercells with their axes oriented along the $[0,1,0]$ and $[0,0,1]$ directions. This set of supercells allowed the analysis of of adsorbed picene molecules at higher coverage, including arrangements having a surface packing and orientation similar to that observed experimentally. In all instances the slabs used in calculations contained five Ag layers, with the top two layers of metal atoms being allowed to relax, and the bottom three layers being kept frozen at their bulk optimized positions. A vacuum region of 26 $\AA$ was employed in the direction perpendicular to the surface.

To aid in the analysis of the features observed in the tunneling measurements, we have also carried out calculations of the anion states using the electron-affinity equation-of-motion MP2(EA - EOM - MP2) method [21] as implemented in the CFOUR code [22] and in conjunction with the cc-pVDZ Gaussian-type orbital basis set [23]. This basis set was chosen to 
prevent the problem of collapse of the anion states onto the continuum [24]. In addition, we also report electron affinities estimated from experimental ionization potentials [25] and from application of the pairing theorem [26]. According to the pairing theorem, the energies of $\pi$ and $\pi^{*}$ orbitals of alternant hydrocarbons are symmetrically located about a reference energy.

\section{Results and Discussion}

\subsection{STM Results}

At sub-monolayer coverage (Fig. 1a), the constant current STM image of a picene molecule on $\operatorname{Ag}(100)$ has a characteristic crescent shape due to the zig-zag arrangement of the benzene rings in the molecular backbone. The apparent approximate size of the molecule is $\sim 1.35 \mathrm{~nm}$ long and $\sim 0.7 \mathrm{~nm}$ wide. The long-axis of the molecule is oriented along the $<010>$ direction of the $\operatorname{Ag}(100)$ surface. At low coverage, the molecules are isolated consistent with repulsive intermolecular interactions. These are likely to be dominated by $\mathrm{H}-\mathrm{H}$ repulsion but could also include dipolar repulsive interactions [27], due to small charge transfer between the surface and the molecules or from Pauli repulsion [28] that overcome the attractive van-derWaals interactions.

Upon increasing the surface coverage to about $0.5 \mathrm{ML}$ (region 1 in Fig. 1b and Fig. 2d), the picene molecules are seen to adopt a partially ordered arrangement with the molecular axes twisted slightly off the original $45^{\circ}$ orientation, and with the molecules stacked along the short axes. We refer to this structure as phase 1 . The intermolecular spacing along the rows is $\sim 0.7-0.8$ $\mathrm{nm}$, while that across the rows is $\sim 1.5 \mathrm{~nm}$. The ordering is not regular, as evidenced by meandering of the molecular displacements along the close-packed rows. Further increase of the coverage completes the monolayer (region 2 in Figs. $1 \mathrm{~b}$ and 1d). In contrast to the low coverage 
where the molecules repel one another (e.g. Fig. 1a), a new phase, denoted phase 2, nucleates within phase 1 and grows as islands (Fig. S1). Moreover, as we will see from the calculations discussed below, about half the molecules in phase 2 are tilted with respect to the surface and bonded to it via one of their edges. As will be discussed later the net intermolecular interactions when increasing the coverage to $1.0 \mathrm{ML}$ are attractive, in contrast to those at or below $0.5 \mathrm{ML}$. The STM images of the full monolayer, display an alternating pattern along the rows (Fig. 1d), implying that the unit cell is comprised of two inequivalent molecules. There is also a subtle modulation of the intermolecular spacing, which doubles the size of the unit cell, with the net dimensions being $v=3 \mathrm{~nm}$ and $u=1.1 \mathrm{~nm}$ (Fig. 1d). Despite being more highly ordered than the phase 1 structure in which the molecules are bound parallel to the surface (Figs. $1 \mathrm{~b}$ and 1d, as well as Fig. 2d), the tilted molecules in the phase 2 structure display small, random deviations from translational periodicity that are apparent in high-resolution images (Fig. 1d).

At still higher coverage, after saturation of phase 2, growth proceeds into the multilayer regime, where locally saturated $3 \mathrm{D}$ islands of two or more layers of picene molecules grow on top of the monolayer (Fig. 1c). Therefore, on the $\operatorname{Ag}(100)$ surface, phase 2 is the most ordered arrangement of molecules, while growth itself proceeds in the Stranski-Krastanov mode [29] where formation of the second layer precedes growth of a three-dimensional film.

Without going into great detail, the growth mode of picene molecules is qualitatively similar on $\mathrm{Cu}(100)$ as seen in Fig. 2. The largely disordered layer of flat-lying molecules (region 1 in Fig. 2a) begins to develop into more highly ordered islands (regions 2 in Fig. 2a). For the $\operatorname{Ag}(111)$ surface, the coexistence of two types of structures is also observed [13], although the lower coverage phase 1 structure is more highly ordered than phase 2 . The electronic properties of the resulting surface structures were probed using scanning tunneling spectroscopy. To 
eliminate the current-induced damage of the adsorbed layers, we have used the technique of Z-V spectroscopy [30], in which the tunneling current is kept constant during the voltage ramp. When the tunneling energy matches the energy of a molecular resonance (i.e., the energy of an occupied or unfilled orbital), the tip retracts to maintain overall a constant tunneling probability. The energies of the electronic states are therefore registered as steps in the height $Z$ of the STM tip relative to the metal surface or as peaks in $d Z / d V$.

As seen in Fig. 3a, for the phase 1 structure of picene on $\operatorname{Ag}(100)$, broad resonances due to anion formation are registered at $1.3,2.1$, and $3.3 \mathrm{eV}$. There is also a sharp peak at $3.5 \mathrm{eV}$ that we attribute to the image potential state of the metal surface. Fig. 3b shows the corresponding results for the phase 2 (monolayer) system. The resonances in phase 2 structure are notably narrower than those in the phase 1 structure, most likely due to reduced electronic coupling of the molecules in the phase 2. Moreover, each of the first two peaks appears to be comprised of two closely spaced features as seen in Figs. 3c and 3d. Using Lorentzian fits (Fig. 3d), the energies of these features are estimated to be 1.55 and $1.69 \mathrm{eV}$ and 2.23 and $2.35 \mathrm{eV}$, respectively. As with the phase 1 structure, the phase 2 structure displays a sharp resonance centered at $3.5 \mathrm{eV}$ (Fig. 3b) associated with the image potential state. In addition, a filled state resonance is observed at $\sim-1.37 \mathrm{eV}$ (Fig. 3e).

\subsection{Experimental analysis of picene epilayers}

As was previously concluded for picene on $\operatorname{Ag}(111)$ [13], the phase 1 structure should not be viewed as a complete monolayer because all molecules in the saturated phase 2 are in direct contact with the metal surface. This is not obvious upon visual inspection of the STM images in Figs 1 and 2, where the molecules adsorbed in going from the phase 1 structure to the phase 2 structure appear to have grown on top of the molecules adsorbed flat on the surface (e.g. 
Fig. 2d). However, both the apparent height measurements and the calculations discussed below support a phase 2 structure with about half of the molecules being tilted with respect to the surface and bound to it edge wise. The STM measurements give an apparent height of $\sim 100 \mathrm{pm}$ (Fig. 1e) for the picene molecules relative to $\operatorname{Ag}(100)$ in phase 1, and a change in the apparent height of $\sim 170 \mathrm{pm}$ between phase 1 and phase 2 (Fig. 1f). The height difference between the top of the phase 2 and the second layer is $\sim 600 \mathrm{pm}$ and that between the second and third layers is 600-700 pm (Fig. 1g). The experimental measurements yield contours of constant tunneling probability rather than the topography, but the two become equivalent if the electronic structure and the dielectric structure of measured objects are comparable. The positions of molecular resonances in the tunneling spectra (discussed below) do not significantly depend on tip-sample distance, which implies that the potential drops primarily across the vacuum gap, which is consistent with a wide gap (low tunneling current) and a dielectric constant of $3-4 \varepsilon_{0}$ characteristic of organic semiconductors. Therefore the apparent height difference of $170 \mathrm{pm}$ in going from the phase 1 to phase 2 is expected to be quite close to the topographic height difference, even though the height of the molecules comprising phase 1 is clearly underestimated by STM topography. The smallest unit-cell dimension of bulk picene is $615.4 \mathrm{pm}$, which is comparable to the separations between the layers after the first full monolayer, but far greater than the $170 \mathrm{pm}$ height difference between the phase 1 and phase 2 structures.

\subsection{Structural analysis from the DFT calculations}

\subsubsection{Phase 1}

In the computational studies, we first examined the adsorption configurations of picene molecules on the $\operatorname{Ag}(100)$ surface at low surface coverage. This was accomplished by placing a single picene molecule in the $(4 \times 4),(6 \times 6)$ and $(8 \times 8)$ supercells with the long axis of the molecule 
taken parallel to the Ag-Ag close contacts. The results of this analysis are summarized in Fig. 4. At low coverage, corresponding to a single picene in the $(8 \times 8)$ surface unit (Fig. 4a), the molecule adsorbs flat on the surface and can assume three different orientations. In one (denoted $\alpha$ ), the molecular axis is parallel to the $[0,0.5,-0.5]$ axis of the $\operatorname{Ag}(100)$ surface, and in another (denoted $\delta$ ), it is diagonally oriented along the $[0,1,0]$ axis. The third orientation (denoted $\gamma$ ) corresponds to an intermediate structure with the axis rotated by about $18^{\circ}$ relative to that defined by the Ag-Ag short distance. For each of these three orientations, we investigated structures with the molecular center initially located above fcc, hollow, or top sites. Panel a) in Fig. 4 indicates the evolution from the initial structures to the optimized structures. For the $\alpha$ and $\delta$ configurations, adsorption at both the fcc and hollow sites is found to lead to stable minima, while for the $\gamma$ configuration only a single stable structure located above the hollow site was determined. The adsorption energy is calculated to be relatively insensitive to the orientation of the molecule on the surface. As a result, at low coverages the adsorbed picene molecules should readily rotate and diffuse on the $\operatorname{Ag}(100)$ surface. Regardless of their positions or orientations, at low coverage the picene molecules are predicted to lie nearly flat on the surface. This is illustrated for $\delta(\mathrm{fcc})$ in the inset panel in Fig. $4 \mathrm{a})$, which provides a side view of the adsorption structure. As seen in this panel, the picene molecule lies flat on the surface at a distance of about $3.0 \AA$ above the metal atoms in the top layer.

The variations with increasing coverage of the calculated adsorption energies for the most stable $\alpha$ and $\delta$ configurations of a picene molecule on the $\operatorname{Ag}(100)$ surface are reported in Fig. 4b). The results were obtained from calculations performed using the (8x8), (6x6) and $(4 \times 4)$ supercells. The calculated adsorption energies decrease with increasing coverage, although for the $\delta$ configuration the decrease is relative moderate, going from $2.47 \mathrm{eV}$ for the $(8 \times 8)$ supercell 
to $2.31 \mathrm{eV}$ for the $(4 \mathrm{x} 4)$ supercell. For the $\alpha$ configuration the adsorption energy drops precipitously, particularly when going from the $(6 \times 6)$ to the $(4 \times 4)$ supercell. Due to the increase of the lateral repulsion between $\mathrm{H}$ atoms on different molecules, the $\alpha$ configuration becomes highly destabilized at higher coverages. These calculations demonstrate that as $0.5 \mathrm{ML}$ coverage is approached, only the $\delta$ adsorption configuration, in which the molecule is oriented "diagonal" along the $[0,1,0]$ direction, would be expected to survive. This result is consistent with the experimental observation for the preferred orientation of picene molecules up to $0.5 \mathrm{ML}$ coverage (Fig, 1a). For the $\delta$ configuration, we considered structures with the long axes of neighboring molecules oriented either perpendicular $(\delta(\mathrm{I})$ in Fig. $4 \mathrm{c}))$ or parallel $(\delta(\mathrm{II})$ and $\delta(\mathrm{III})$ in Fig 4c)) to one another. Essentially the same binding energy is obtained for these two arrangements at this coverage.

We also examined the sensitivity of the adsorption configuration and binding energy of picene molecules to the surface as the lateral separation between the molecules is decreased. This was done using the $8 \times 5$ and $7 \times 5$ surface supercells (see Figs. 5a) and c)) oriented along the $[0,1,0]$ and $[0,0,1]$ directions, respectively, and containing two rows of molecules. The separation between the centers of the rows is $16.4 \AA$ for the $8 \times 5$ supercell and $14.4 \AA$ for the $7 \times 5$ supercell. As seen from the side views of the resulting configurations depicted in panels a) and b) of Fig. 5 the molecules at these coverages remain flat on the surface and have comparable binding energies of 2.49 and $2.46 \mathrm{eV}$.

\subsubsection{Phase 2}

We examined next the adsorption of picene molecule at coverages greater than 0.5 ML. This has been done for the case of $(7 \times 5)$ supercell by increasing the number of molecules in each 
row from two to three [see panels c) and d) in Fig. 5c]. As shown in these figures, upon optimization, the structure changes from one with all molecules lying flat on the surface to structures in which either about a third of all molecules are tilted (see Fig. 5c) or all of them are tilted by some degree (see Fig. 5d) with respect to the surface. These two arrangements are essentially isoenergetic with adsorption energies of about $1.9 \mathrm{eV} /$ molecule, significantly smaller than that $(2.5 \mathrm{eV} /$ molecule $)$ for phase 1 . These findings suggest that a simple increase in the number of molecules in the first layer from two to three for each row in $(7 \times 5)$ supercell (see Figs. $5 \mathrm{c}$ and $5 \mathrm{~d}$ ) is highly unfavorable and these higher coverage arrangements were not considered further.

Additionally, we analyzed whether picene molecules could adsorb lying flat on top of the molecules already adsorbed flat on the surface. This has been done for the case of the $(7 \times 5)$ supercell containing a total of twelve molecules distributed equally in two rows (each containing two layers). Calculations reveal (for a typical case see Fig. S2) that such arrangements are unstable with respect to those in which some of the molecules are tilted and some are bonded edgewise to the surface. For this reason, in the ensuing discussion we focus on structures in which some molecules are bonded directly to the metal surface but are tilted with respect to the surface.

The most stable arrangement for phase 2 has all the molecules beyond $0.5 \mathrm{ML}$ tilted with respect to the monolayer and located in between the molecules of the first layer, as shown in panels e) and f) of Fig. 5. The average binding energy of all molecules, both flat and tilted, is $2.16 \mathrm{eV}$, for both the $7 \times 5$ and $8 \times 5$ supercells. The most tilted molecules are less strongly adsorbed than the nearly flat-lying molecules, with binding energies of 1.86 and $1.84 \mathrm{eV}$ for $7 \mathrm{x} 5$ and $8 \times 5$ supercells, respectively. However, we note that these adsorption energies are higher 
than the cohesive energy between picene molecules in the crystal. For picene crystal with a monoclinic structure [31], we calculate a cohesive energy of $1.76 \mathrm{eV}$ per molecule. (For the optimized crystal, our calculated lattice parameters of 8.449,6.112, and $13.454 \AA$ agree to within $1.5 \%$, with the experimental values of $8.480,6.154$, and $13.515 \AA[31])$.

Calculations carried out with only the tilted molecules on the surface, give a binding energy of about $1.2 \mathrm{eV} /$ molecule, which leads us to conclude that for the phase 2 structure, about $0.9 \mathrm{eV}$ of the binding energy per molecule of the tilted molecules comes from interactions with the molecules bound flat (or nearly so) on the surface. This molecule-molecule interaction is expected to be dominated by dispersion and electrostatic contributions. While our calculations indicate that the average binding energy per molecule is reduced in going from phase 1 to phase 2, Hasegawa and co-workers [13], reached the opposite conclusion concerning the relative stabilities of the two phases. In this case, however, phase 2 would precede the formation of phase 1 , which is not the case as seen from the results of our experiments

Overall, the results presented in Figs. 5 and S2 provide strong support that phase 2 is a mixture of molecules lying nearly flat on the surface and molecules that are tilted with respect to the surface plane, in agreement with the experimental findings. The most notable conclusion is that the intermolecular interactions contribute as much as $0.9 \mathrm{eV} /$ molecule to the binding, allowing the titled configuration to be energetically preferred over a hypothetical flat lying bilayer.

\subsection{Analysis of tunneling spectroscopy measurements}

We now turn to the analysis of the tunneling spectra of the phase 2 structure (shown in Fig. 3b-f). Table 1 summarizes the energies of the anion states of picene (referenced relative to 
the ground state anion) obtained from EA-EOM-MP2 [21] calculations using the cc-pVDZ basis set, from use of the pairing theorem [26] and from experiment, together with our assignments. The calculated results are for the gas-phase molecule while the experimental results are from the present measures of picene monolayer on $\operatorname{Ag}(100)$.

\section{Table 1}

Relative energies (eV) of the $\pi^{*}$ anion states of picene

Gas-phase picene

STS of Phase 2

Picene/ $\operatorname{Ag}(100)$ EOM-MP2

Pairing theorem ${ }^{\mathrm{c}}$

$0.0,0.14^{2}$

$0.0,0.04$

$0.0,0.13$

$0.67,0.79^{\mathrm{a}}$

0.66

0.82

1.43

1.30

1.52

$(1.80)^{\mathrm{b}}$

1.80

1.74

2.55

2.41

2.38

\footnotetext{
${ }^{\mathrm{a}}$ The doublet structures in the STS features were determined by a fitting procedure described in the text.

${ }^{\mathrm{b}}$ Although the STS measurements do not display a distinct feature near $1.80 \mathrm{eV}$, such a feature could be "hidden" on the low-energy side of the intense feature associated with the image potential state.

${ }^{\mathrm{c}}$ These results are derived from the experimental IPs of picene reported in [25].
}

For phase 2 picene $/ \operatorname{Ag}(100)$ the relative energies of molecular resonances are $0.14,0.67$, 0.79, $1.43 \mathrm{eV}$, and $2.55 \mathrm{eV}$ (based on Fig. 3). Comparison of the EOM and experimental results leads to the following assignment of the experimentally observed features: The first broad resonance (overlapping features at 0 and $0.14 \mathrm{eV}$ ) derives from the ground and first excited anions states, which are predicted to be nearly degenerate, the second broad resonance (overlapping features at 0.67 and $0.79 \mathrm{eV}$ ), is due to the third anion state, and the 1.43 and 2.55 
$\mathrm{eV}$ features to the fourth and sixth anion states, respectively. The calculations place the fifth anion state $1.80 \mathrm{eV}$ above the ground state, and although experiment does not display a clear feature at this energy, it could be hidden under the low-energy shoulder of the image potential state. Finally, the predictions of the pairing theory are very close to those of the EOM calculations, providing further support for these assignments.

The major issue with the above assignment concerns the $(0.67,0.79 \mathrm{eV})$ doublet observed experimentally, while the calculations predict only a single anion state in this energy range. We believe that it is unlikely that the experimentally observed splitting derives from picene molecules in different environments. A more likely possibility is that the splitting results from the interactions between picene molecules. If this is the case, then the lower energy doublet ( 0 and $0.14 \mathrm{eV}$ ) could also have as its origin the splitting caused by interactions between picene molecules.

Overall good agreement is found between the computed energy differences for the gasphase molecule and the corresponding STS results for the phase 2 system. This indicates that there is an approximately uniform shift in the energies of the various $\pi^{*}$ anion states of picene in going from the gas-phase molecule to the phase 2 adsorbed species. The anion states of the phase 1 structure are stabilized by additional $200-300 \mathrm{meV}$ compared to titled molecules in the phase 2 structure, presumably due to the stronger interaction with the metal surface.

Assuming that the broad peak at $\sim-1.3 \mathrm{eV}$ in the STS measurements (Fig. 3e) corresponds to electron ejection from the HOMO, the HOMO-LUMO gap for picene molecules in the phase 2 structure is then $2.9 \mathrm{eV}$, while the experimental IP - EA value for the gas-phase molecule is $7.0 \mathrm{eV}$. The $4 \mathrm{eV}$ reduction in the HOMO-LUMO gap in going from the gas-phase 
molecule to the phase 2 structure, implies that the on-site Coulomb potential is reduced by about $2.0 \mathrm{eV}$ due to screening by the neighboring molecules and the metal surface.

A similar conclusion is reached by estimating the extent of the screening from the shift in the LUMO in going from the gas phase molecule to the phase 2 structure. The first EA of picene in the gas phase is $0.6 \mathrm{eV}$ (referenced to vacuum) [1] while the energy of the first anion state of the phase 2 structure of picene on $\mathrm{Ag}(100)$ is $1.55 \mathrm{eV}$ relative to the Femi level. Since the work function of $\mathrm{Ag}(100)$ is $\sim 4 \mathrm{eV}$, we conclude that the anion state is stabilized by $1.8 \mathrm{eV}$ in going from the gas-phase molecule to the phase 2 structure. Thus the reduction in the $\mathrm{U}$ value due to screening as deduced from the shift of the LUMO level is consistent with that determined from the change in the HOMO-LUMO gap. The work-function of $4 \mathrm{eV}$ was estimated from the position of the image potential state $(3.5 \mathrm{eV})$, the binding energy of which is $\sim 0.5 \mathrm{eV}$ below vacuum level on $\operatorname{Ag}(100)[33]$.

Interestingly, the band-gap of a crystalline picene is $\sim 3.3 \mathrm{eV}$ close to that determined here for phase 2 picene $/ \operatorname{Ag}(100)$, which indicates that the screening due to the metal substrate and surrounding molecules in adsorbed picene is comparable to that in the picene crystal. This immediately suggests that despite close-proximity to the metal surface, Coulomb screening in polyaromatic molecular systems remains weak. Even for an electronic bandwidth (W) of $\sim 0.5$ $\mathrm{eV}$, the $\mathrm{U}_{\mathrm{scr}} / \mathrm{W}>1$, making the emergence of Mott-insulating state quite likely in doped picene. In prior studies, the Coulomb repulsion has been assumed to be $\sim 1-2 \mathrm{eV}$ in picene [7], but the models show a metal-insulator transition around $0.8 \mathrm{eV}$ [34]. At the same time, the regime analyzed in Ref. [11], where there is suppression of the Mott insulating state and formation of $3 / 4$ filled bands in 3e-doped picene, necessitates $\mathrm{U}$ values of $>5 \mathrm{eV}$. The screening in either bulk or adsorbed forms is too strong to allow such cross-over between $1 / 2$ filled and two $3 / 4$ filled bands, at 
least within the assumed approximations. This situation may be different however, in a condensed phase adsorbed on insulators, where there would be no effects of image screening due to proximity to metallic substrate.

We now examine more closely the resonance structure in the STS measurements of picene on $\operatorname{Ag}(100)$ under different coverages. Fig. 3a reports the spectrum for the phase 1 structure which corresponds to flat-lying molecules. Figures $3 \mathrm{~b}$ and $3 \mathrm{~d}$ report the spectra for the phase 2 structure and Fig. 3c reports the spectrum due to a mixture of phase 1 and phase 2 structures. Fig. $3 \mathrm{f}$ demonstrates how the spectrum in Fig $3 \mathrm{c}$ arises as a superposition of those from phase 1 and phase 2 structures. However, as we discussed above, the detailed origin of splitting in Fig. 3d (and its effect on spectra in Fig. 3c) will need further investigation, as the magnitude of the splitting of the peaks at $\sim 1.5 \mathrm{eV}$ is too large to be explained by the neardegeneracy of LUMO/LUMO+1 states, while the splitting of the peaks at $\sim 2.25 \mathrm{eV}$ is too small to be accounted for by $\mathrm{LUMO}+2 / \mathrm{LUMO}+3$ splitting.

\subsection{Picene epilayer doped with potassium}

Much of the controversy regarding K-doped picene and related polyaromatic hydrocarbons [6,34] has to do with the absence of well-defined single crystals and instability of such materials in ambient environment, which significantly hinder electron transport measurements on any kind of alkali doped material. Thin films would be very beneficial in this regard as would be the prospect of direct characterization of K-doping, or even the hypothesized superconducting state, with tunneling spectroscopy. To this end, we attempted to dope the wellcharacterized films with potassium. To avoid deposition of hot $\mathrm{K}$ atoms directly onto the molecular film, we first deposited $\mathrm{K}$ onto $\operatorname{Ag}(100)$ and then overgrew picene. $\mathrm{K}$ would not 
diffuse into the bulk of the Ag crystal under these conditions. We further confirmed that the work-function of the surface is lowered by at least $2 \mathrm{eV}$ by $\mathrm{K}$ deposition [35].

As clearly seen in Figs. 6a and 6b, the quality of the films that form on K-doped $\operatorname{Ag}(100)$ is far inferior to that observed on the clean metal surface. In particular, there is an almost complete lack of long-range ordering as evidenced by the Fourier transform of the STM image (Fig. 6c). STM topography reveals multiple holes on the surface, which based on the constant current topography represent single molecular layers (Fig. 6d). The other characteristic feature is that the molecules surrounding the holes have slightly higher apparent topography (Fig. 6d). The cause of the observed disorder is not clear. However, one possibility would be the strong electrostatic interactions involving negatively charged picene molecules and $\mathrm{K}^{+}$ions. We could also be observing a chemical reaction between potassium and picene as suggested by the calorimetry study of Ref. [6].

\section{Conclusions}

In conclusion, picene is shown to be able to form two types of structures on noble metal surfaces. In the lower coverage phase, denoted phase 1, the molecules lie flat on the surface, and in the higher coverage phase, denoted phase 2 , half of the molecules lie nearly flat on the surface and the other half lie tilted about $30 \mathrm{deg}$. with respect to the plane of the surface plane. Our calculations show that a significant portion of the stability of phase 2 comes from attractive interactions between the tilted and flat lying molecules. Scanning tunneling microscopy measurements show that the HOMO-LUMO gap is reduced from $7 \mathrm{eV}$ to about $3 \mathrm{eV}$ for $\operatorname{Ag}(100)$, with the latter value being close to that of the bulk crystal. The sizable reduction in the on-site Coulomb repulsion upon formation of the monolayer makes it an interesting molecular model system for strongly correlated electronic ground states upon doping. Finally, we attempted 
to carry out K-doping of picene monolayer and found that in the coverage regime tested, $\mathrm{K}$ doping caused significant disorder, most likely caused by electrostatic interactions. We believe that scanning probe microscopy with molecular resolution may provide critical insight into the structure, chemistry and electronic states of the potassium doped polyaromatic hydrocarbons, particularly if bulk-like arrangements of molecules can be stabilized in ultrathin structures. Here, we showed that even a monolayer of picene on certain metal surfaces may satisfy this criterion and approach bulk-like "herringbone" arrangement of molecules as well as electrostatic screening comparable to bulk values. Further effort combining tunneling spectroscopy and epitaxially grown films is likely to produce deeper insight into doping of polyaromatic hydrocarbons, with direct implications for their presumed phase-transitions.

\section{Acknowledgments}

SK, JW, PM. Experiments were carried out at the Center for Nanophase Materials Sciences, sponsored at the Oak Ridge National Laboratory, by the Division of User Facilities, Basic Energy Sciences, U.S. Department of Energy. KDJ and KA acknowledge support from the National Science Foundation through grant CHE-1362334. Some of the calculations were carried out at the University of Pittsburgh's Center for Simulation and Modeling. 


\section{References}

[1] NIST Chemistry WebBook, http://webbook.nist.gov/chemistry/.

[2] H. Okamoto, N. Kawasaki, Y. Kaji, Y. Kubozono, A. Fujiwara, M. Yamaji, J. Am. Chem. Soc. 130 (2008) 10470-10471.

[3] E.A. Silinsh, Organic Molecular Crystals, Springer, Berlin, 1980.

[4] R. Mitsuhashi, Y. Suzuki, Y. Yamanari, H. Mitamura, T. Kambe, N. Ikeda, et al., Nature. 464 (2010) 76-79.

[5] Y. Kubozono, H. Goto, T. Jabuchi, T. Yokoya, T. Kambe, Y. Sakai, et al., Phys. C-Supercond. Its Appl. 514 (2015) 199-205.

[6] S. Heguri, M. Kobayashi, K. Tanigaki, Phys. Rev. B. 92 (2015) 014502.

[7] G. Giovannetti, M. Capone, Phys. Rev. B. 83 (2011) 134508.

[8] K. Teranishi, X. He, Y. Sakai, M. Izumi, H. Goto, R. Eguchi, et al., Phys. Rev. B. 87 (2013) 060505.

[9] G.A. Artioli, L. Malavasi, J. Mater. Chem. C. 2 (2014) 1577-1584.

[10] D. Hillesheim, K. Gofryk, A.S. Sefat, Nov. Supercond. Mater. 1 (2015). http://www.degruyter.com/view/j/nsm.2013.1.issue-1/nsm-2015-0001/nsm-20150001.xml (accessed September 23, 2015).

[11] T. Dutta, S. Mazumdar, Phys. Rev. B. 89 (2014) 245129.

[12] S.S. Naghavi, M. Fabrizio, T. Qin, E. Tosatti, Phys. Rev. B. 88 (2013) 115106.

[13] Y. Yoshida, H.-H. Yang, H.-S. Huang, S.-Y. Guan, S. Yanagisawa, T. Yokosuka, et al., J. Chem. Phys. 141 (2014) 114701.

[14] J.B. Neaton, M.S. Hybertsen, S.G. Louie, Phys. Rev. Lett. 97 (2006) 216405.

[15] Y. Wang, R. Yamachika, A. Wachowiak, M. Grobis, K.H. Khoo, D.-H. Lee, et al., Phys. Rev. Lett. 99 (2007) 086402.

[16] J. VandeVondele, M. Krack, F. Mohamed, M. Parrinello, T. Chassaing, J. Hutter, Comput. Phys. Commun. 167 (2005) 103-128.

[17] CP2K. Version 2.5. CP2K is freely available from www.cp2k.org., n.d.

[18] J.P. Perdew, K. Burke, M. Ernzerhof, Phys. Rev. Lett. 77 (1996) 3865-3868.

[19] S. Grimme, J. Antony, S. Ehrlich, H. Krieg, J. Chem. Phys. 132 (2010) 154104.

[20] J. VandeVondele, J. Hutter, J. Chem. Phys. 127 (2007) 114105.

[21] J.F. Stanton, J. Gauss, J. Chem. Phys. 103 (1995) 1064-1076.

[22] CFOUR, a quantum chemical program package written by J.F. Stanton, J. Gauss, M.E. Harding, P.G. Szalay, with contributions from A.A. Auer, R.J. Bartlett, U. Benedikt, C. Berger, D.E. Bernholdt, Y.J. Bomble, L. Cheng, O. Christiansen, M. Heckert, O. Heun, C. Huber, T.-C. Jagau, D. Jonsson, J. Jusélius, K. Klein, W.J. Lauderdale, F. Lipparini, D.A. Matthews, T. Metzroth, L.A. Mück, D.P. O'Neill, D.R. Price, E. Prochnow, C. Puzzarini, K. Ruud, F. Schiffmann, W. Schwalbach, C. Simmons, S. Stopkowicz, A. Tajti, J. Vázquez, F. Wang, J.D. Watts and the integral packages MOLECULE(J. Almlöf and P.R. Taylor), PROPS (P.R. Taylor), ABACUS (T. Helgaker, H.J. Aa. Jensen, P. Jørgensen, and J. Olsen), and ECP routines by A. V. Mitin and C. van Wüllen.

[23] T.H.D. Jr, J. Chem. Phys. 90 (1989) 1007-1023.

[24] M.F. Falcetta, K.D. Jordan, J. Phys. Chem. 94 (1990) 5666-5669. 
[25] R. Boschi, E. Clar, W. Schmidt, J. Chem. Phys. 60 (1974) 4406-4418.

[26] C.A. Coulson, G.S. Rushbrooke, Proc Camb. Phil Soc. 36 (1940) 193.

[27] J. Fraxedas, S. García-Gil, S. Monturet, N. Lorente, I. Fernández-Torrente, K.J. Franke, et al., J. Phys. Chem. C. 115 (2011) 18640-18648.

[28] X. Crispin, V. Geskin, A. Crispin, J. Cornil, R. Lazzaroni, W.R. Salaneck, et al., J. Am. Chem. Soc. 124 (2002) 8131-8141.

[29] S.R. Forrest, Chem. Rev. 97 (1997) 1793-1896.

[30] D.B. Dougherty, P. Maksymovych, J. Lee, M. Feng, H. Petek, J.T. Yates, Phys. Rev. B. 76 (2007) 125428.

[31] A. De, R. Ghosh, S. Roychowdhury, P. Roychowdhury, Acta Crystallogr. C. 41 (1985) 907909.

[32] B.M. Savoie, N.E. Jackson, T.J. Marks, M.A. Ratner, Phys. Chem. Chem. Phys. 15 (2013) 4538-4547.

[33] K. Giesen, F. Hage, F.J. Himpsel, H.J. Riess, W. Steinmann, Phys. Rev. B. 35 (1987) 971974.

[34] A. Ruff, M. Sing, R. Claessen, H. Lee, M. Tomić, H.O. Jeschke, et al., Phys. Rev. Lett. 110 (2013) 216403.

[35] D. Heskett, T.M. Wong, A.J. Smith, W.R. Graham, N.J. DiNardo, E.W. Plummer, J. Vac. Sci. Technol. B. 7 (1989) 915-918.

[36] S. Laurent, kmeans++ Matlab routine, n.d.

http://www.mathworks.com/matlabcentral/fileexchange/28804-k-means++. 


\section{Figures}
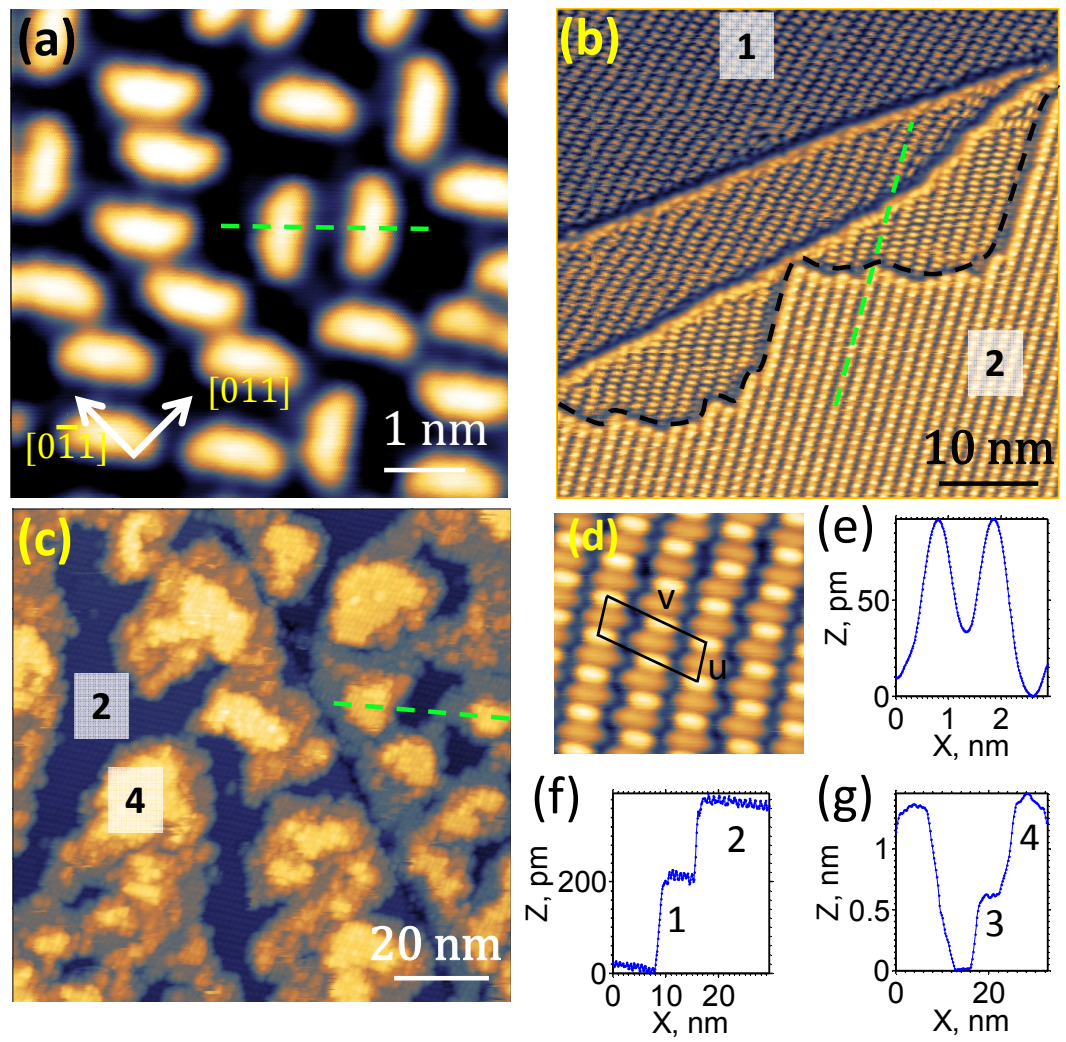

Fig. 1. Constant current STM images of picene molecules on $\operatorname{Ag}(100)$ surface: (a) at low coverage (acquired at bias $\mathrm{V}=1 \mathrm{~V}$ and current setpoint $\mathrm{I}=30 \mathrm{pA}$ ); (b) at higher coverage where both phase 1 (region 1) and phase 2 (region 2) coexist $(\mathrm{U}=0.7 \mathrm{~V}, \mathrm{I}=140 \mathrm{pA})$; (c) at still higher coverage where the phase 2 structure is fully saturated (region 2) and multilayer islands up to nominally three layers begin to nucleate $(\mathrm{U}=3.2 \mathrm{~V}, \mathrm{I}=100 \mathrm{pA})$. (d) A close-up STM image of the phase 2 system, with unit-cell vectors $\mathrm{u}$ and $v$ described in the text $(\mathrm{U}=0.7 \mathrm{~V}, \mathrm{I}=$ 140 pA). (e-f) Topographic line profiles along green dashed lines in images (a-c), correspondingly. Numbers in the images and line-profiles correspond to the nominal thickness of the layers. 

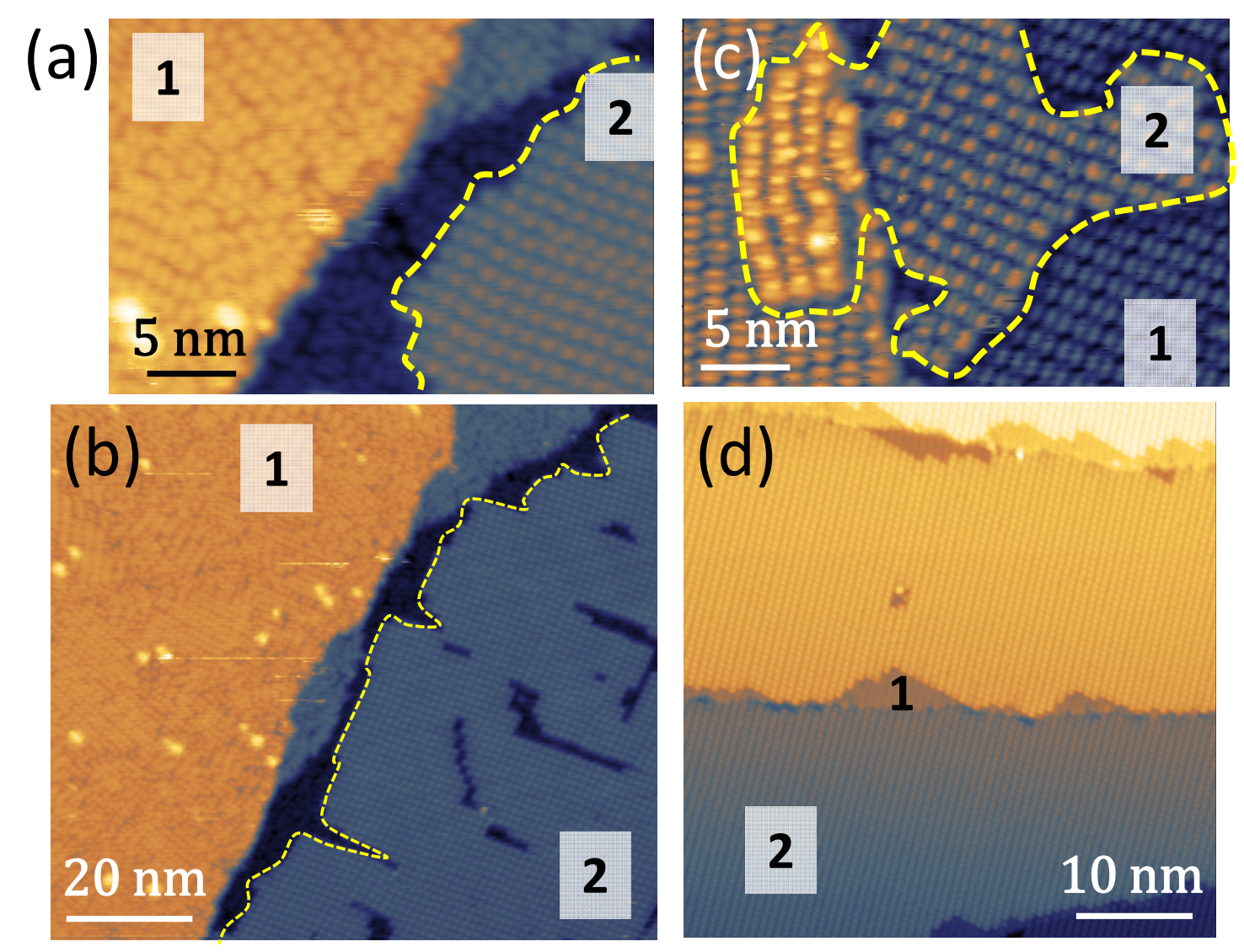

Fig. 2. Constant current STM images of coexisting phases 1 and 2 on $\mathrm{Cu}(100)$ surface $(\mathrm{a}, \mathrm{b})(\mathrm{U}=$ $0.3 \mathrm{~V}, \mathrm{I}=30 \mathrm{pA}), \operatorname{Ag}(111)$ surface $(\mathrm{c})(\mathrm{U}=1.2 \mathrm{~V}, \mathrm{I}=20 \mathrm{pA})$, and $\operatorname{Ag}(100)$ surface $(\mathrm{d})(\mathrm{U}=0.5$ $\mathrm{V}, \mathrm{I}=30 \mathrm{pA})$. The phase 1 and phase 2 regions are marked in each case. 

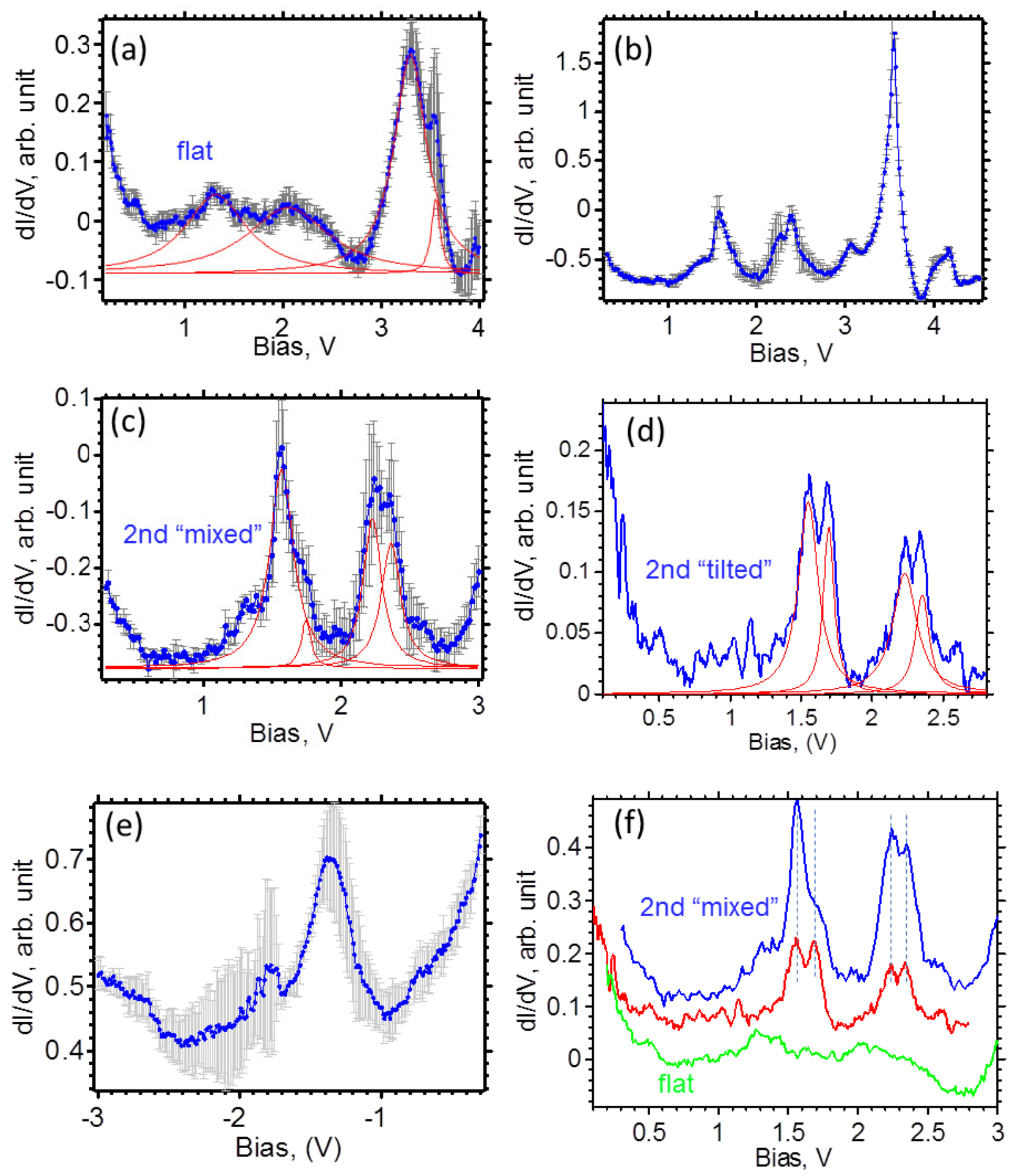

Fig. 3. Tunneling spectroscopy of empty electronic states of picene on $\operatorname{Ag}(100)$. Blue is the average of several (2-20) individual I-V curves; grey is the error bar corresponding to one standard deviation; red is the fit to the observed peaks using a Lorentzian function. (a) phase 1 ; (b) phase 2. (c) Mixed phase 1 and phase 2, (d) low-energy anion states of phase 2 
picene/ $\operatorname{Ag}(100)$ with observable fines structure within each of the first two main peaks and the accompanying fit with four Lorentzians. (e) Filled state region of the phase 2 picene on $\operatorname{Ag}(100)$. (f) Overlay of three kinds of spectra in (a), (c) and (d) that reveals their relationship to each other. All spectra were acquired at $77 \mathrm{~K}$ using $\mathrm{Z}-\mathrm{V}$ spectroscopic mode with a constant-current set-point of $\sim 100 \mathrm{pA}$. Note that the spectra show $\mathrm{dI} / \mathrm{dV}$, acquired as the first harmonic of voltage-modulated tunneling current with the AC amplitude of 5-10 $\mathrm{mV}$ and frequency of 650 $\mathrm{Hz}$. 


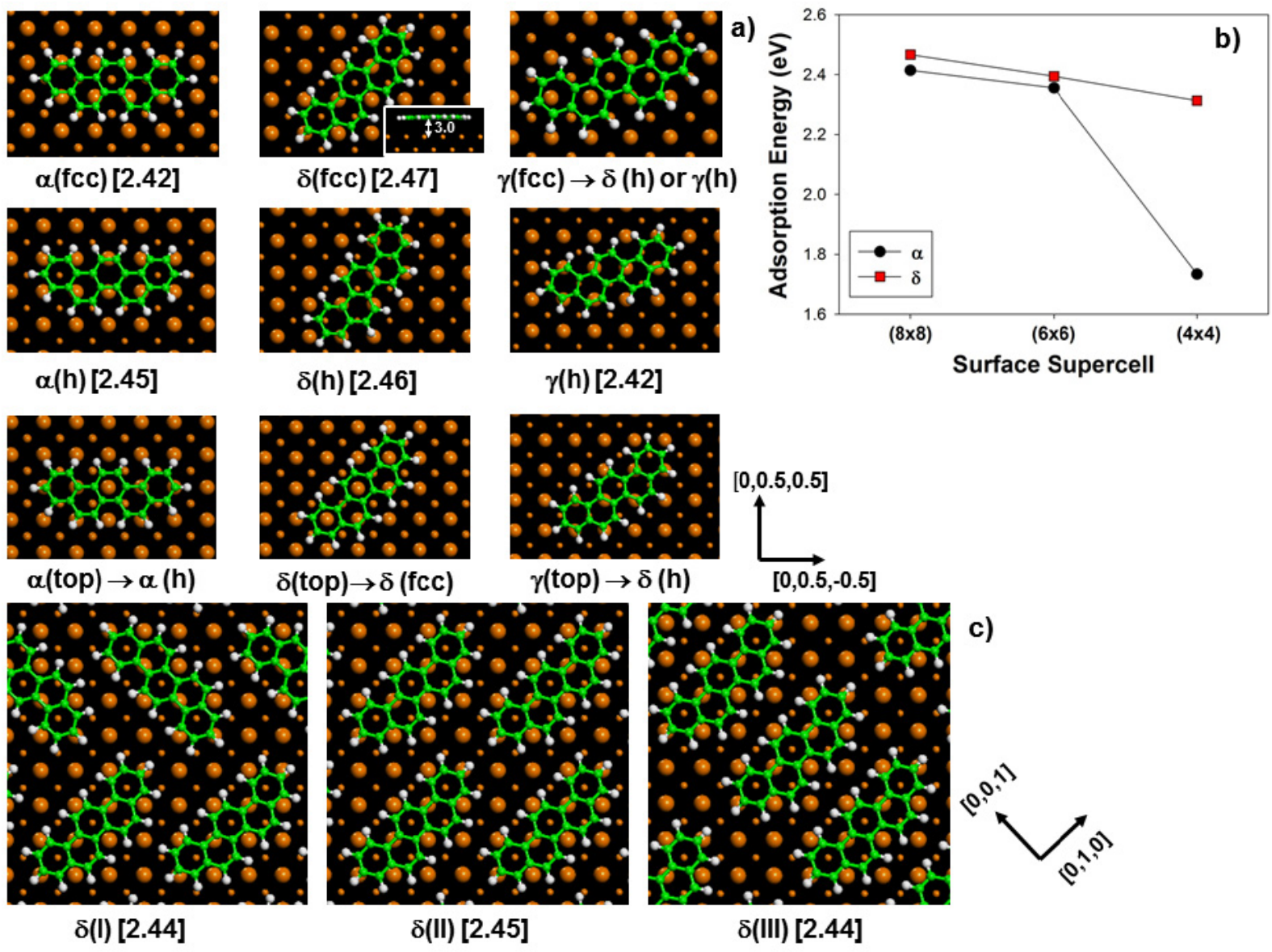

Fig.4. Adsorption configurations of picene in the dilute to phase 1 regime as determined by DFT calculations. In all these cases the molecules are lying flat on the surface. For the stable adsorption configurations the binding energies in $\mathrm{eV}$ of the final optimized structures (shown in the figures) are given in parentheses. For unstable configurations the evolution of the depicted structure from the initial (shown in the figure) to final structures is indicated. The fcc, hollow (h) and top labels used refer to the specific locations on the surface of the middle of picene molecule. The $\alpha, \delta$ and $\gamma$ arrangements are defined in text. 


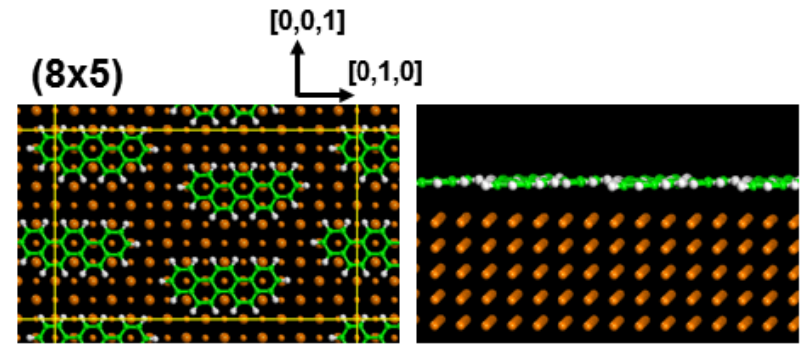

a) $\delta$ (fcc) [2.49]

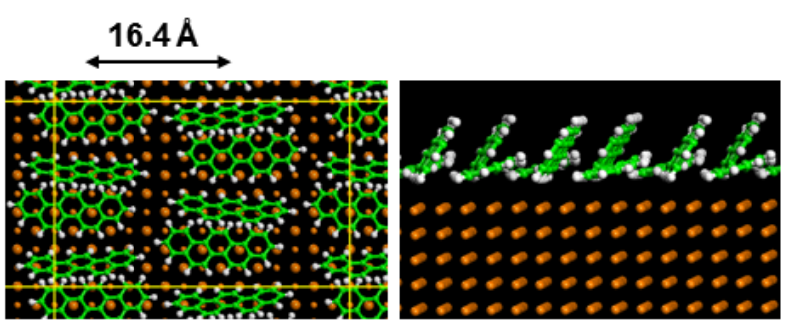

f) $\mathrm{Ph}$ (II) [2.16]

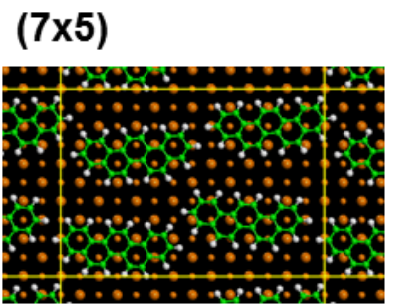

b) $\delta$ (fcc) [2.46]

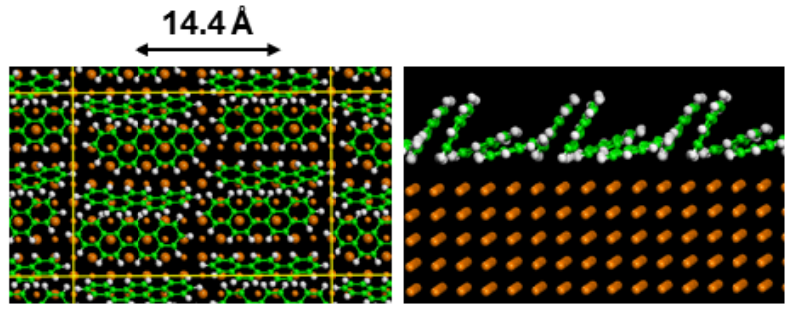

e) $\mathrm{Ph} 2$ (I) [2.16]

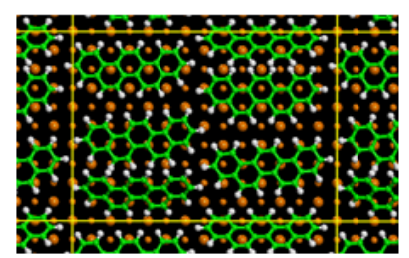

c) $\delta$ (fcc+hcp) [1.94]
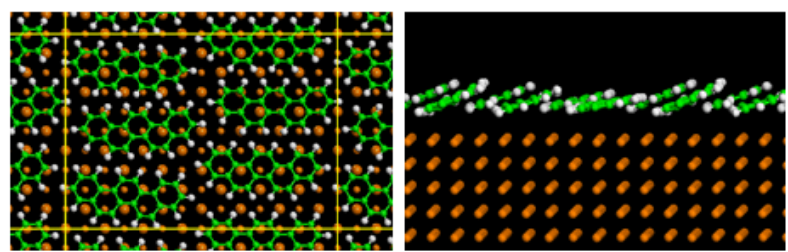

d) $\delta$ (fcc+hcp) [1.97]

Fig. 5. Adsorption configurations of picene in phase 1 at $0.5 \mathrm{ML}$ (panels (a) and (b)), at intermediate coverages above $0.5 \mathrm{ML}$ (panels (c) and (d)) and in phase 2 at full ML coverage ( panels (e) and (f)) as determined by DFT calculations. The number of picene molecules per supercell is four ((a),(b)), six ((c), (d)), and eight ((e),(f)). The supercells used in calculations are indicated in the figures. The acronyms Ph2(I) and Ph2(II) refer to molecular configurations of picene in phase 2 in $(7 \times 5)$ and (8x5) supercells, respectively. The other acronyms are explained in the main text. 

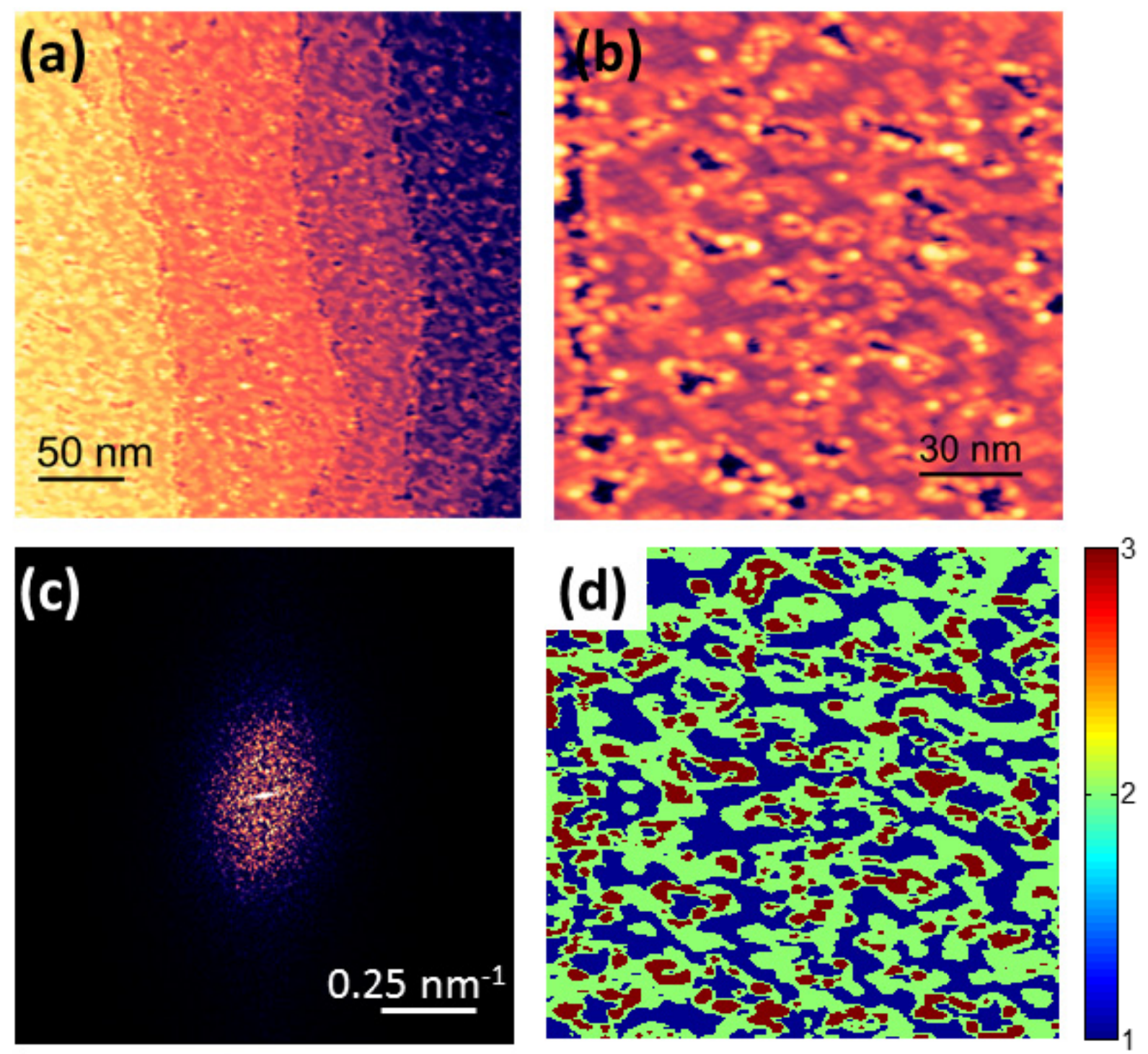

Fig. 6. STM images of picene grown on top of a K-doped $\operatorname{Ag}(111)$ surface. (a,b) Large and small-scale constant-current topography images of typical structures. (c) 2D-FFT of the image in (b) that shows lack of apparent long-range order. (d) k-means cluster transform applied to (b). Here the constant-current heights from the STM image are partitioned into three clusters using the k-means++ clustering algorithm [36]. The colors in the image correspond to the cluster number. There are three characteristic features in this image. These are: the flat-lying picene 
molecules in cluster 1 (possibly phase 1), and slightly higher molecules in cluster 2 (possibly tilted as in phase 1) and the bare surface in cluster 3 . 
Graphical abstract

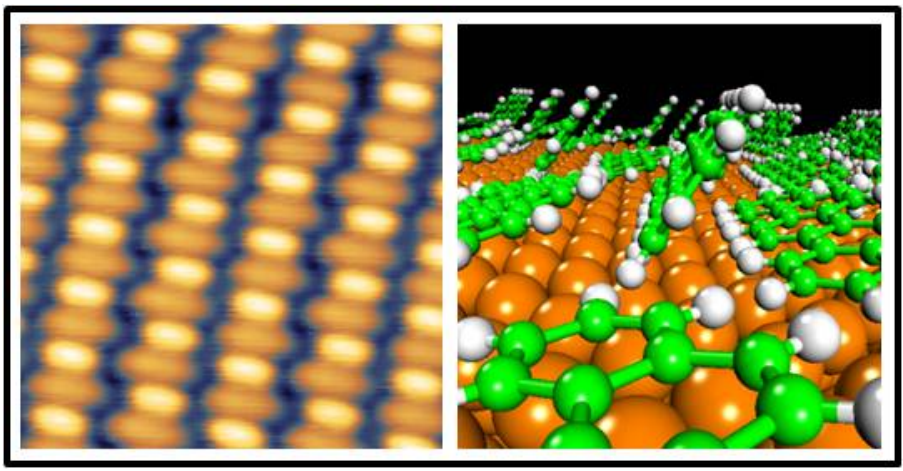

